\title{
The measurement of suspension rheology
}

\author{
E. J. Hinch $\dagger$
}

Department of Applied Mathematics and Theoretical Physics, University of Cambridge, Centre for Mathematical Sciences, Wilberforce Road, Cambridge CB3 0WA, UK

In the following featured article, Boyer, Pouliquen \& Guazzelli (J. Fluid Mech., this issue, vol. 686, 2011, pp 5-25) measure the normal stresses in a suspension of non-colloidal rigid spheres. They use the classical rod-climbing experiment, except that for interesting reasons the free surface near to the rotating rod does not rise but dips down. Careful techniques reveal that the normal stresses occur only above a volume concentration of $22 \%$. Over a period of hours the measurements drift, typical of many observations of suspensions. This is due to particles slowly migrating away from the rotating rod. A model of the migration gives good predictions of the observed changes.

\section{Rheology}

Suspensions of particles have a complex rheology. Most suspensions have nonviscous forces which drive the suspension to a well-defined rest state when the flow is turned off, e.g. colloidal suspensions of hard particles have Brownian, electrical double-layer and van der Waal's forces, while soft particles can have capillary forces, elasticity or bending stiffness.

The suspensions studied by Boyer, Pouliquen \& Guazzelli (2011), hereafter BPG, have non-colloidal hard spheres. There is therefore no relaxation to a rest state. Without a relaxation process with an intrinsic time scale, the stress must be linear in the magnitude of the instantaneous strain rate, and this has important consequences. Note that the stress can still change in time, but only over times measured in units of the inverse strain rate. Note that the stress tensor need not be linear as a tensor in the strain-rate tensor, i.e. each component having the same coefficient of proportionality; in fact, in the flow studied by BPG it is not linear as a tensor.

The flow studied by BPG is a wide-gap circular Couette flow with a stationary outer cylinder and a rotating inner cylinder, which is called a 'rod' in the case of the wide gap. Circular Couette flow is one example of a general class of so-called 'viscometric' flows in which one layer of fluid continually slides over another. In these locally simple shear flows, the directions of the flow, velocity gradient and vorticity are all orthogonal, say respectively in the $x, y$ and $z$ directions; the flow is then locally $\boldsymbol{u}=(\gamma y, 0,0)$ with shear rate $\gamma$. The rheology of viscometric flows is characterized

\section{$†$ Email address for correspondence: ejh1@cam.ac.uk}


by three universal functions, a viscosity $\mu$ and two so-called normal stress differences $N_{1}$ and $N_{2}$. The viscosity characterizes the tangential friction components of the stress tensor, i.e. $\sigma_{y x}=\sigma_{x y}=\mu \gamma$, and these components do work. The two normal stress differences describe the non-isotropic nature of the diagonal elements of the stress tensor, with $N_{1}=\sigma_{x x}-\sigma_{y y}$ and $N_{2}=\sigma_{y y}-\sigma_{z z}$, and these components of the stress do no work.

A typical suspension with a relaxation process to a rest state will have a shearthinning viscosity, a positive $N_{1}$ and a relatively small $N_{2}$; all independent of the sign of the shear rate. The positive $N_{1}$ can be described as a tension in the streamlines, and comes from a microstructure being stretched in the direction of the straining and then rotated a little by the vorticity into the direction of the flow. At low shear rates, $N_{1}$ and $N_{2}$ must be quadratic in the shear rate, and for thermodynamic and stability reasons $N_{1}$ must be positive.

In the absence of a relaxation process and a natural rest state, BPG's non-colloidal suspension of hard spheres has a quite different rheology. The viscosity in steady shear will be independent of the shear-rate, i.e. has no shear-thinning. The two normal stress differences are linear in the shear rate, or to be more precise linear in the modulus of the shear rate, because they must be independent of the sign of the shear rate (direction of the flow). Lacking a relaxation process, there is no thermodynamic reason for $N_{1}$ to be positive. In fact, in these non-colloidal suspensions of hard spheres $N_{1}$ is small and probably negative, while now $N_{2}$ is large and certainly negative. These normal stresses come from the repulsion between two spheres as they approach one another in the shear flow, pushing one another away fairly equally in the flow and the flow-gradient directions. The fairly equal push in these two directions makes $N_{1}$ small, while the lack of serious repulsion in the vorticity direction makes $N_{2}$ large and negative. These normal stress differences can be described as a tension in the vortex lines.

\section{Rod climbing experiments}

When a rotating rod is placed in a container of water, the water adjacent to the rod also rotates, and then through centrifugal forces is thrown outwards. The free surface is thus depressed near to the rod. When a rotating rod is placed in a container of an elastic liquid or a typical suspension, those liquids with positive $N_{1}$ and small $N_{2}$, the liquid does the opposite and climbs the rod. It is the tension from $N_{1}$ in the curved streamlines near to the rod which produces a hoop stress squeezing the liquid inwards. The free surfaces rises until the additional hydrostatic pressure pushing outwards balances the hoop stress squeezing inwards. When a rotating rod is placed in a container of BPG's suspension of non-colloidal hard spheres, the free surfaces dips downs. With no significant $N_{1}$, it is the negative $N_{2}$ which puts the vortex lines in tension. The free surface is pulled down by the tension in the vortex lines, which is higher near to the rotating rod because the shear rates are higher there.

The classical perturbation analysis of rod-climbing by Beavers \& Joseph (1975) finds the free surface is displaced upwards proportional to a certain combination of normal stresses, $\left(N_{2}+0.25 N_{1}\right)$, and varies in the radial direction as $r^{-4}$. This is the radial variation of the normal stresses, which are proportional to the square of the shear rate, which is the radial derivative of the $1 / r$ flow. BPG's suspension of non-colloidal rigid spheres have normal stresses proportional to the shear rate, not the 
square, and so the displacement of the free surface now varies as $r^{-2}$. There is also a consequential change in the combination of the normal stresses to $\left(N_{2}+0.5 N_{1}\right)$. The slower $r^{-2}$ radial variation usefully presents more to be measured by experiments.

The method used by BPG to measure the radial profile of the vertical displacement of the free surface is to project a series of parallel white lines onto the surface from a projector angled to the vertical, see their figures 1 and 2. Vertical displacements of the free surface then cause the white lines to deviate horizontally. Testing the approach on an inverted rigid cone showed that surface displacements of around $5 \mathrm{~mm}$ could be measured to an accuracy of $0.1 \mathrm{~mm}$, which was less than the diameter of the particles. The observed radial profile was first corrected by subtracting small inertial effects. Then observations within a couple of capillary lengths of the inner and outer cylinders were discarded. Finally fitting the expected $r^{-2}$ profile then yields the combination of the normal stresses $\left(N_{2}+0.5 N_{1}\right)$ with very good accuracy.

Figure 6 in BPG's paper gives the important results for how the normal stresses depend on the volume concentration of the suspension. They have plotted the nondimensional normal stresses $\alpha=N / \mu \gamma$. The new results have less experimental uncertainty and scatter compared with previous measurements of rod-climbing by Zarraga, Hill \& Leighton (1999) and Singh \& Nott (2003). With the improved accuracy, one sees clearly for the first time that the normal stresses are only nonzero once the volume fraction exceeds $\phi_{c}=0.22$. Above this critical value, the normal stresses seem to increase linearly with the concentration, $\alpha_{2}+0.5 \alpha_{1}=-1.4\left(\phi-\phi_{c}\right)$. There is no difference between the results with the two different sizes of spheres.

\section{Particle migration}

While the rod rotates on the time scale of a second, the free surface displacement drifts on the time scale of an hour, see BPG's figure 7(a). There is a corresponding drift in the torque interpreted as an apparent viscosity, see figure $9(a)$. I believe that such slow drifts were first reported by Acrivos's student F. Gadala-Maria in his 1979 $\mathrm{PhD}$ thesis. The phenomenon was explained as particle migration by a later student of Acrivos, D. T. Leighton, in his 1985 PhD thesis. BPG measured the radial variation of the concentration of particles in their experiments after a long time, showing in their figure $8(a)$ migration away from the higher shear rates next to the inner rod.

For a first model of the slow migration of particles across streamlines, Leighton \& Acrivos (1987) suggested that there would be fluxes of particles proportional to the gradient of concentration and the gradient of the shear rate, both due to more particles hitting an individual particle on the side up the gradient. Later Nott \& Brady (1994) suggested an alternative 'suspension balance model' in which the migration velocity is driven by a divergence in the 'particle stresses'. That the particle stresses are proportional to the shear rate and depend on the particle concentration, the resulting migration velocity will have contributions proportional to the gradients in both, as in Acrivos's original model. Recently Nott, Guazzelli \& Pouliquen (2011) have questioned the origin of the particle stress.

BPG use a suspension-balance model, or two-phase fluid model, to calculate the particle migration. The particle phase satisfies conservation of mass and momentum in their equations (4.3), along with a drag law (4.4) between the particle and fluid phases. These first parts of the model are standard. Modelling the 'particle stress' (4.7), which drives the particle migration, requires assumptions for values of the normalized normal stress differences $\lambda_{1}$ and $\lambda_{2}$ and for the form of the 'normal viscosity' $\hat{\eta}_{n}$. BPG use their measured rheology to suggest (4.12) for the latter. Numerical solution of the 
migration model gives a series of good predictions, for the drift in the radial variation of the particle concentration in figure 8 , for the drift in the measurement of the torque (apparent viscosity) in figure $9(a)$ and for the drift in the negative rod-climbing in figure 7 .

\section{Future}

While the suspension model of the particle migration successfully predicts several different observations, they are related observations, and so should perhaps count as fewer in number than the number of assumptions. It would therefore be good to see in the future the model employed to predict behaviour in some additional new flows. One can also look forward to a clearer understanding of the nature of the particle stress (Nott et al. 2011).

BPG's rod-climbing experiments measure a single combination $\left(N_{2}+0.5 N_{1}\right)$ of the normal stress differences. Previous rod-climbing experiments by Zarraga et al. (1999) and Singh \& Nott (2003) had more scatter in their data. However, these two groups also used a parallel-plate rheometer to measure $\left(N_{1}-N_{2}\right)$, and a coneand-plate rheometer to measure $N_{1}$. In a second paper, Couturier et al. (2011) have used Tanner's tilted trough technique to measure $N_{2}$ in isolation. They find (see their figure 6) that this normal stress probably vanishes at a slightly lower volume fraction of $17 \%$.

The curious behaviour of elastic liquids with a large positive $N_{1}$ can be understood in a number of flows as the result of tension in the streamlines, e.g. rod-climbing, the migration of particles to the centre line of a pipe flow, the vertical alignment of falling rods, the stabilization of jets and a purely elastic Taylor-Couette instability. It would be interesting to consider the dynamical consequences of tension in vortex lines for flows of non-colloidal suspensions.

\section{References}

Beavers, G. S. \& Joseph, D. D. 1975 The rotating-rod viscometer. J. Fluid Mech. 69, 475-512.

Boyer, F., Pouliquen, O. \& Guazzelli, E. 2011 Dense suspensions in rotating-rod flows: normal stresses and particle migration. J. Fluid Mech. 686, 5-25.

Couturier, E., Boyer, F., Pouliquen, O. \& Guazzelli, E. 2011 Suspensions in a tilted trough: second normal stress difference. J. Fluid Mech. 686, 26-39.

GADAlA-MARiA, F. 1979 The rheology of concentrated suspensions. PhD thesis, Stanford University.

Leighton, D. T. 1985 The shear-induced migration of particles in concentrated suspensions. $\mathrm{PhD}$ thesis, Stanford University.

LEIGHTON, D. T. \& ACRIVOS, 1987 Measurement of shear-induced self-diffusion in concentrated suspensions of spheres. J. Fluid Mech. 177, 109-131.

NotT, P. R. \& BRADY, J. F. 1994 Pressure-driven flows of suspensions: simulation and theory. J. Fluid Mech. 275, 157-199.

Nott, P. R., Guazzelli, E. \& Pouliquen, O. 2011 The suspension model revisited. Phys. Fluids 23, 043304 .

SingH, A. \& NotT, P. R. 2003 Experimental measurements of the normal stresses in sheared Stokesian suspensions. J. Fluid Mech. 490, 293-320.

Zarraga, I. E., Hill, D. A. \& Leighton, D. T. 1999 The characterization of the total stress of concentrated suspensions of non-colloidal spheres in Newtonian fluids. J. Rheol. 44, 185-220. 